\title{
The Effect of Prenatal Factors on Crown Dimensions
}

\author{
S. M. GARN, ${ }^{1}$ R. H. OSBORNE ${ }^{2}$ AND K. D. MCCABE ${ }^{3}$ \\ ${ }^{1}$ Center for Human Growth and Development, University of Michigan, Ann Arbor, \\ Michigan 48104, U.S.A.; ' Department of Anthropology, University of Wisconsin, \\ Madison, Wisconsin 53706; and ${ }^{3}$ The Collaborative Perinatal Project of the \\ National Institute of Neurological and Communicative Diseases and Stroke. \\ Bethesda, Maryland 20014
}

\begin{abstract}
KEY WORDS Tooth size - Crown size - Mesiodistal - Buccolingual . Maternal effects . Gestational effects . Historical trends
\end{abstract}

\begin{abstract}
As shown in 870 white participants in the National Collaborative Perinatal Project (NCPP), maternal health status during pregnancy and birth size are systematically related to mesiodistal and buccolingual crown dimensions of $\mathrm{I} 1, \mathrm{I} 2, \mathrm{dc}, \mathrm{dm} 1, \mathrm{dm} 2$ and M1. Maternal diabetes, maternal hypothyroidism and large size at birth are associated with larger maxillary and mandibular teeth in white children. Conversely, deciduous and permanent crown diameters are diminished in maternal hypertension, and in low birthweight and small birth-length conditions. These findings suggest that maternal and fetal (or gestational) determinants of both deciduous and permanent tooth crown dimensions may account for as much as half of crown-size variability with major implications to population comparisons and historical odontometric differences and trends.
\end{abstract}

The extent to which crown sizes of either deciduous or permanent teeth may be affected in the course of prenatal development is at present unknown, yet quantitative information is essential to all studies that employ crown-dimensions and to all students of dental development.

The possibility that the mesiodistal and buccolingual crown diameters may be increased or decreased by events that occur before birth has bearing on all population comparisons, since both population similarities and population differences might be exag. gerated by prenatal developmental events. Moreover, long-term trends in crown-size, conventionally viewed in evolutionary perspec. tive, might actually reflect alterations in the prenatal milieu and differential survival rath. er than actual changes in gene-frequency.

If, indeed, crown sizes can be altered by the maternal environment during odontogenesis, or by differential rates of fetal development, then many traditional approaches merit reexamination. Twin similarities in crown-size and morphology might well be the results of the shared intrauterine environment and the stress of multiple pregnancies. Even postnatal sibling resemblances in crown-size and morphology might also be inflated by similarities in placental size and efficiency and by maternal health status. In turn, intraindividual crown-size correlations, similarities between isomeres and antimeres and even odontometric "fields" might well derive more from specific intrauterine events at critical times in odontogenesis and less from genetic programming.

As compared with these concerns, the supporting evidence is indeed meagre, in large part because the problem has scarcely been broached in systematic fashion. What appears in textbooks of oral pathology is largely anecdotal, and without extensive samples, lacking prospective studies or even retrospective designs. The "hardest" relevant data in the literature pertain to crown sizes in chromosomal abnormalities including Downs Syndrome (47 $\mathrm{G}+$ ) Klinefelter's syndrome (XXY), the Super-male (XYY) etc. (cf. Cohen et al., '70; Garn et al., '70 '71; Cohen et al., '77). Next in order of "hardness," insofar as sample sizes and inferences go, are evidences of diminished 
crown size in cleft-palate syndromes (Osorio, '70; Garn, '77; Dijkman, '78). In these several examples, odontogenesis is certainly disturbed, crown-sizes are distinctly altered, but it is by no means certain that the effects are strictly prenatal in timing or the products of the intrauterine environment. Similar restrictions pertain to the teeth in the maternal rubella syndrome (Evans, '44, '47).

More pertinent to the problem than studies of dysmorphogenesis or the maternal rubella syndrome are the findings of Bailit and Sung ('68). In this study, based on ratings of dental maturation on 289 boys and girls attending a Teamster Union dental clinic, there were statistically-significant negative correlations between rates of dental developments and birth weight, birth order and maternal age. Their findings on 289 children from 89 families suggest that specific maternal effects and prenatal developmental rates may together have bearing on crown size as well as on calcification and formation rates extending through postnatal time.

In the present study, therefore, we have attempted to resolve the primary problem through the use of a unique data base, a truly large sample and through the use of carefullyselected and critically-compared subsamples of boys and girls who experienced clearly-defined prenatal problems or extremes of gestation length and size at birth. The results, based upon automated-caliper (ALICE) measurements of both deciduous and permanent teeth in 2,149 children followed from before birth through the early school years and beyond do indeed suggest that a variety of maternal states and several gestational variables relate to alterations in the crown size dimensions of both deciduous and permanent teeth. As shown by comparisons both within the study sample and by comparison with an independent normative series it now seems unquestionable that alterations in the rates of prenatal dental development may affect crown sizes to a surprising degree. In consequence, the results bear on all of our notions concerning crown size and its various determinants.

\section{MATERIALS AND METHODS}

This study is based upon mesiodistal and buccolingual crown-size measurements of a special subsample of the 1963-1966 birth cohort of participants in the Collaborative Perinatal Project of the National Institute of
Neurological and Communicative Disorders and Stress (NINCDS). The children were studied at the time of their 7- to 8-year examination, along with a selected group of clinically. normal siblings. Subjects derived from six cooperating institutions - in Baltimore, Maryland; Buffalo, New York; Philadelphia, Pennsylvania; Providence, Rhode Island; Richmond, Virginia and Portland, Oregon. For a description of the NCPP project, see Niswander and Gordon ('72).

More than half of the total was selected on the basis of maternal gestational history, birth size and gestation length, or both. The series therefore included children of diabetic mothers, children of hypothyroid mothers, children of hypertensive mothers, low-birthweight neonates and high-birthweight neonates, etc. Clinically-normal children were included for comparison since adequate single-source odontometric standards for both deciduous and permanent teeth did not exist at the time the project was conceived.

At each cooperating institution wax-bite impressions and alginate impressions ("molds") were taken of each subject and plaster casts were then made as soon as practicable. All casts were trimmed at the University of Wisconsin and conventional mesiodistal and buccolingual crown size diameters were taken on all available deciduous and permanent teeth using an automated caliper designed at the University of Washington. This automated caliper, designated by the acronym "ALICE," employed a Helios needle-point caliper in the RUR series with $0.05 \mathrm{~mm}$ readout. Replicate measurements on every fifteenth cast showed that measuring accuracy fell within the $\pm 0.15 \mathrm{~mm}$ limits characteristic of this type of equipment.

The odontometric measurements supervised by Dr. Lassi Alvesalo were edited and then merged on tape with other NCPP data. Data analyses were then completed at the Center for Human Growth at the University of Michigan after further editing and merging with tapes from the NINCDS Collaborative Perinatal Project (NCPP). On the basis of sample sizes and completeness of data maximum attention was given to maternal pregnancy conditions (diabetes, hypothyroidism and hypertension) and to two gestational variables (birth weight and gestation length) defined in terms of the total complement of medically-normal singleton births in the NINCDS Collaborative Perinatal Project. 


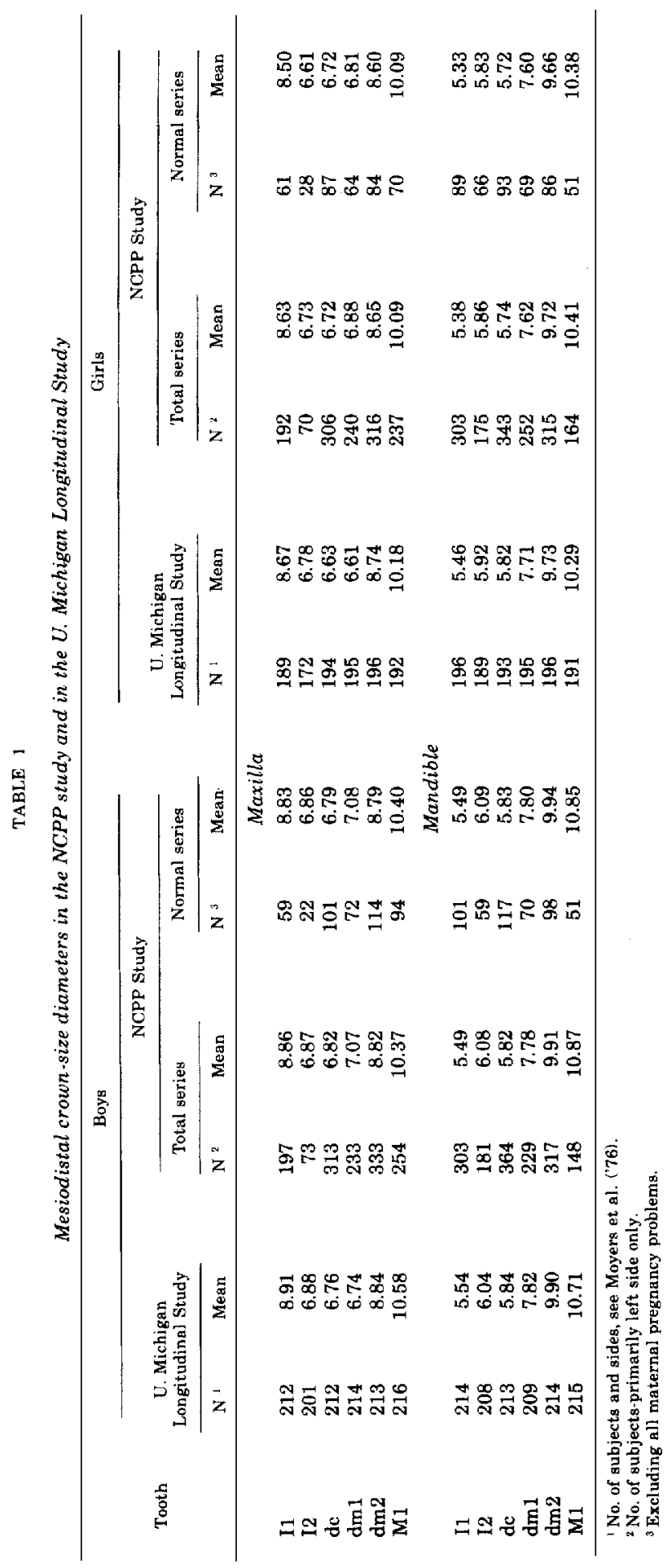


As mentioned, there was legitimate question as to appropriate norms or standards for the odontometric measurements of these children, since published series (Forsyth, Fels, etc.) differ in crown sizes somewhat. Accordingly, three sets of standards were generated-two internal to the study and one external. The internal norms included (a) all children in the special study-including the medical and gestational abnormalities and (b) the clinically-normal group alone. The external norms comprised the University of Michigan Longitudinal series, derived from the University of Michigan School (cf. Moyers et al., '76; Garn and Bailey, '77).

As shown in table 1, where maxillary and mandibular mesiodistal crown-size diameters are compared for the NCPP series and the University of Michigan series, the results are remarkably similar for both permanent and deciduous teeth for white children, i.e., of European ancestry. Despite differences in sampling, differences in measuring equipment (OPTOCOM vs. ALICE) and differences in measuring technique, the close agreement among the data bases attests to the quality of the NCPP data despite their diverse geographic origins and subsamples.

It will be noted that subject Ns for individual teeth fall below the sample size originally stated. This is true because only data on white boys and girls are here included, and because of the age composition of the NCPP sample (which moves into the age of the mixed dentition). Accordingly only six teeth in each jaw are considered in this study, i.e., I1, I2, dc, $\mathrm{dm} 1, \mathrm{dm} 2$ and M1. In some comparisons, only dc (the deciduous canine) is considered, affording 619 maxillary teeth and 707 mandibular teeth for white participants in the NCPP study.

Moreover, despite the large subject $\mathrm{N}$ to begin with, the wide age range considered and tooth loss due to caries together reduce sample sizes for specific deciduous and permanent teeth for many of the birth conditions considered. Accordingly, and in order to simplify data analyses and graphic presentations, sexspecific and tooth-specific normalized $T$ scores or normalized Z scores (Abramowitz and Stegun, '70) were computer-calculated against the distributions for the total series. In this way, data on boys and girls could be pooled and comparisons made of the effect of various maternal gestational conditions on the mesiodistal and buccolingual crown dimensions.
Finally, with tooth sizes for individuals uniformly expressed as normalized $\mathrm{T}$ scores or $\mathrm{Z}$ scores relative to the total series it was then possible to generate and to display crown-size pattern profiles (Garn, '77). This approach, which we have extensively used for crown dimensions and other variables also allows pooling of sexes and affords the opportunity to ascertain whether there are patterned differences in crown-size dimensions.

The present report is designed to summarize lengthy and detailed differences in crown sizes between the various maternal-health subgroups and the gestational subgroups in the simplest fashion yet with sufficient detail for professional workers.

\section{FINDINGS}

In the first step of actual data analysis both mesiodistal and buccolingual crown-size means and standard deviations were calculated for the total NCPP dental series including all maternal and gestational abnormalities - and for the clinically-normal subsample and then compared with comparable crown-size measurements for the University of Michigan Longitudinal Study. Levels of agreement prove to be so high as to allow virtual interchange of odontometric means for 12 teeth (6 in each jaw). Accordingly, the total NCPP sample of 870 white girls and boys could be used as race-specific standard (to maximize subject Ns) or the clinically-normal subsample could be used as the normative group. Indeed, for all practical purposes the University of Michigan Longitudinal Series could be employed as well.

In the next step the various maternal health conditions (hypothyroidism, diabetes, hypertension) and the several gestational variables (gestation-length, birth-size etc.) were considered. Analysis was accomplished tooth by tooth for all deciduous and permanent teeth described-I1, I2, dc, dm1, dm2 and M1. The metric values (i.e., raw scores) were converted into normalized $Z$ scores so as to simpli. fy pooled-sex as well as sex-specific comparisons and comparisons involving different combinations and numbers of deciduous or permanent teeth. The results, shown in tables 2 and 3 for (a) permanent teeth, (b) deciduous teeth, (c) maxillary teeth, (d) mandibular teeth, (e) boys alone, (f) girls alone and (g) combined-sex groupings all follow the same general trends. That is, the children of hypothyroid mothers and diabetic mothers evi- 


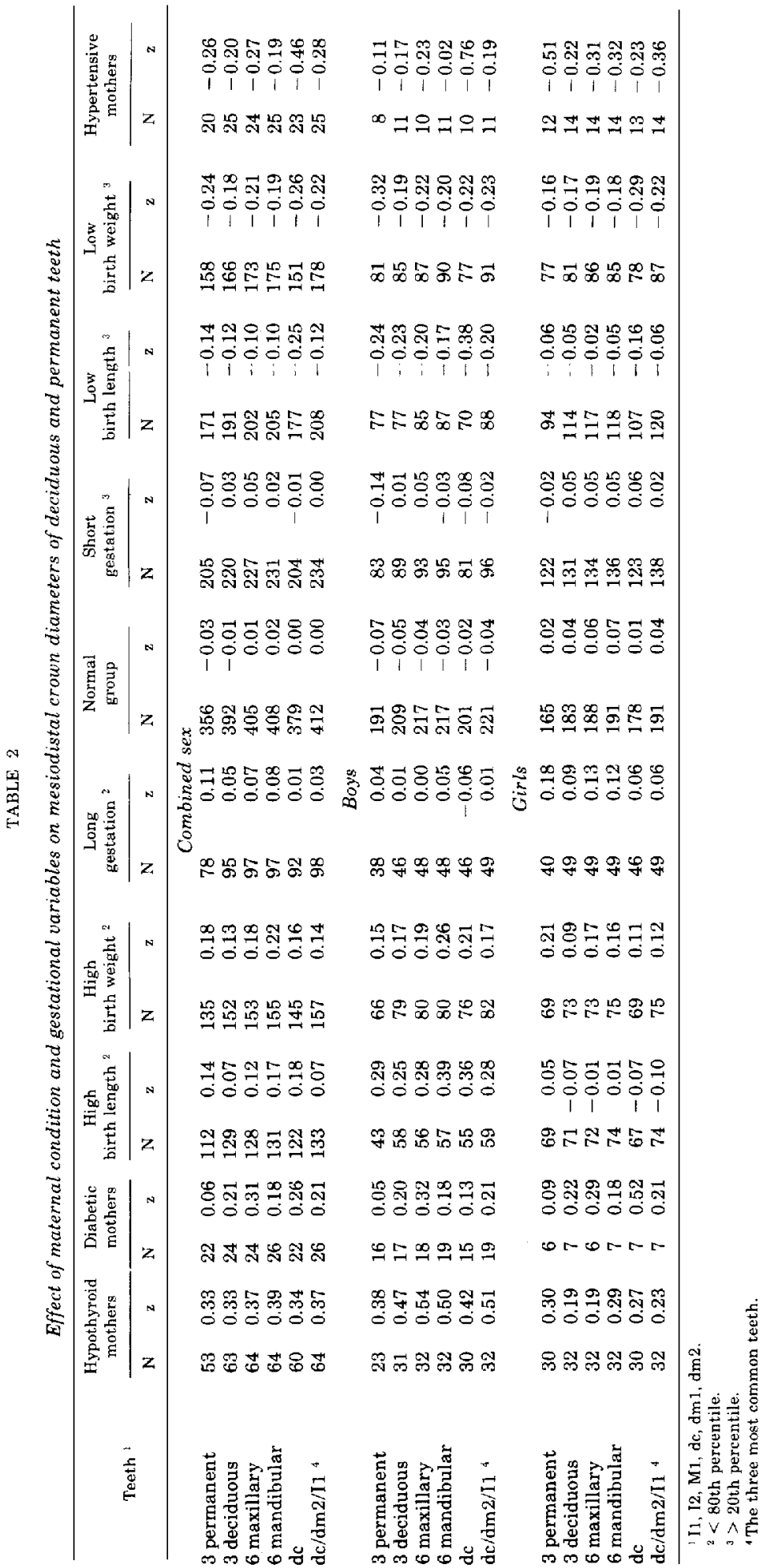




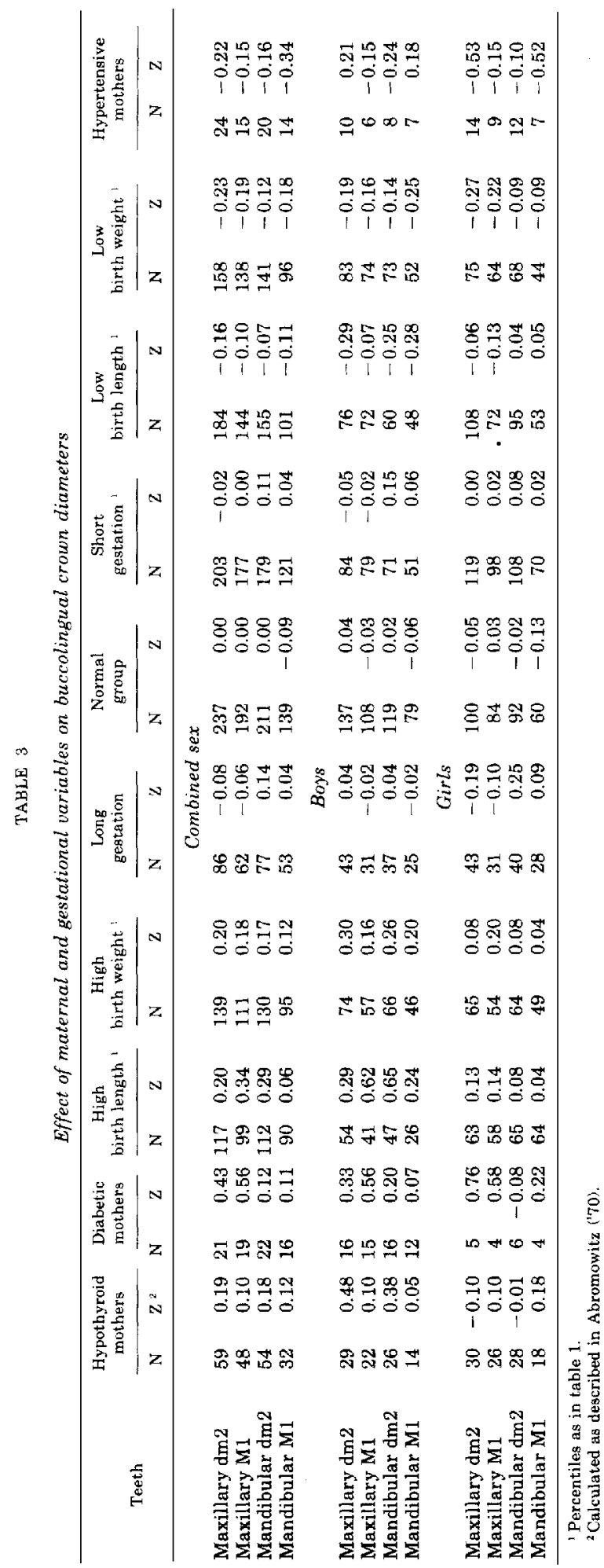




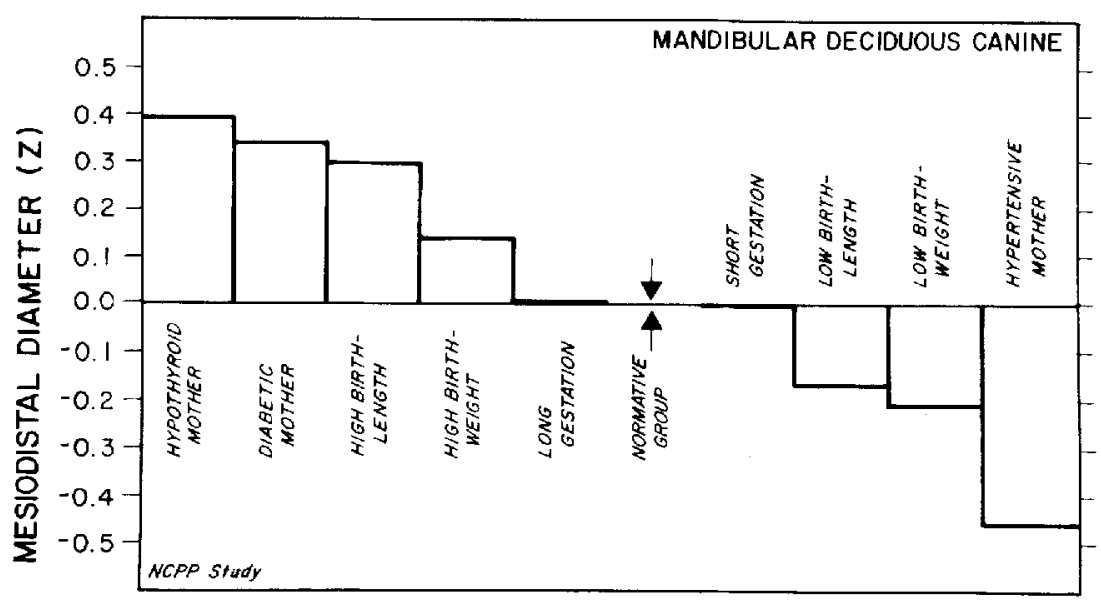

Fig. 1 Comparative sizes of the mandibular deciduous canine in those white boys and girls representing ten different maternal and gestational conditions as compared to the normative group of 210 . As shown, there are systematic effects of maternal hypothyroidism during pregnancy, maternal diabetes, maternal hypertension, birth size and birth weight on the size of this representative tooth. Results are virtually identical whether the normative group alone is used as the dimensional reference or the total NCPP dental sample or the University of Michigan Longitudinal Series.

dence systematically greater crown sizes whereas low birth-length and low birthweight children and the children of hypertensive mothers are associated with systematically diminished odontometric dimensions. This progression is evident in the order of arrangement of maternal conditions and gestational variables as given in the tables. The use of different combinations of teeth, i.e., permanent or deciduous or maxillary or mandibular, or the three most common teeth $(\mathrm{dc}, \mathrm{dm} 2, \mathrm{I} 2)$ does not alter the relative effects or the arrangement of maternal conditions and gestational variables. No test of significance is given here, since mesiodistal and buccolingual crown-size dimensions are all positively correlated as we have previously shown. However sign tests and t-tests can be used to suggest the significance of the differences especially of those involving conditions and gestation length. (Since the $Z$ scores are calculated with reference to the total (NCPP) series, tests of significance allow larger subject $\mathrm{Ns}$ than shown for the normal group alone.)

The findings for different combinations of deciduous and permanent and maxillary and mandibular teeth are perhaps best exemplified for the mandibular deciduous canine alone. This tooth, most often present on an individual basis in the sample, and hence employed for this purpose, exactly follows the order of crown-size enlargement and crownsize reduction shown in detail in table 2 and figure 1. Thus, in left-to-right arrangement there is a diminution in crown sizes. At the extreme left of figure 1 , the deciduous canine crowns average $+0.4 \mathrm{Z}$ for children of hypothyroid mothers with the children of diabetic mothers and for those of large size at birth following in relative order of diminution. At the right hand side of the same figure, deciduous canine size is decreased with the largest decrease observed in the children of hypertensive mothers. Overall, the span of relative tooth sizes from the children of hypothyroid mothers to the children of hypertensive mothers encompasses nearly a full standard deviation (i.e., $1 \mathrm{Z}$ score).

Following the tabular and graphic analyses just described, crown-size pattern-profiles were generated for each of the maternal and gestational conditions mentioned, using sexappropriate and tooth-specific normalized $\mathrm{Z}$ scores calculated in terms of the NCPP sample as a whole. Of these nine maternal and gestational conditions five are now presented for consideration. In each case, the maternal or gestational condition is indicated by the use of solid lines ( $\longrightarrow$ ), the reference or comparison population is designated by dashed lines (- - - ), and the total NCPP series (or for all practical purposes the University of Michigan Longitudinal Series) is the base line or reference line corresponding to a $\mathrm{Z}$-score value of 0.0 .

Turning to the first such maternal condi- 


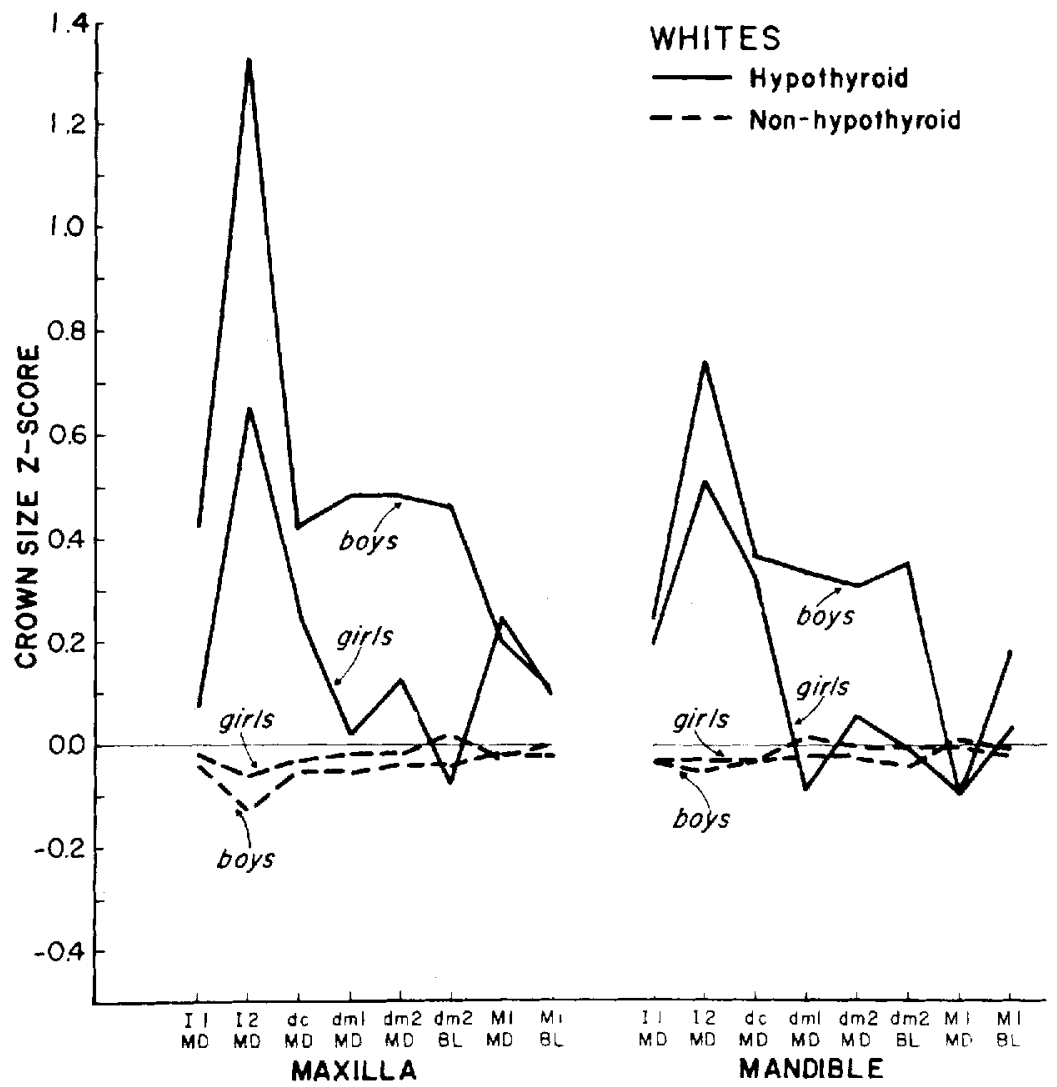

Fig. 2 Crown-size pattern-profiles of deciduous and permanent teeth of 58 children of hypothyroid mothers (-) as compared with 650 sons and daughters of mothers without hypothyroidism during preg. nancy (-- - - ) both in reference to the NCPP total-sample norm. As shown the boys and girls whose mothers were diagnosed as hypothyroid prior to/or during the period of gestation have systematically larger teeth in both the maxilla and the mandible. The mesiodistal diameter of the lateral incisors is most affected. Overall, the children of hypothyroid mothers average 0.3 standard deviations above odontometric expectancy.

tion (maternal hypothyroidism), the patternprofile clearly distinguishes the crown-sizes of 58 children of hypothyroid mothers (solid lines), 650 children of non-hypothyroid mothers (dashed line) and the total white series (870). For both mesiodistal and buccolingual dimensions and for nearly all teeth the children of hypothyroid mothers have larger than normal crown dimensions, particularly so the mesiodistal diameter of both maxillary and mandibular lateral incisors. In this graphic example there is a systematic difference between the two sexes in that the boys rather than the girls seem to be most affected (fig. 2).

For progeny of 20 diabetic mothers shown in figure 3 as against 687 of non-diabetic mothers and for the NCPP sample as a whole, maternal diabetes during pregnancy appears to be associated with elevated crown dimension, the more so if the sex-specific $\mathrm{Z}$ scores are pooled. Maternal diabetes, for these cases, is not associated with systematically greater birth weights. Accordingly, the systematically-increased deciduous and permanent crown dimensions are not reflections of prolonged gestation or diabetic "giantism."

Turning to the birth-weight groupings (as in fig. 4) and birth-length groupings (as in fig. 5) the systematic relationships between birth size and crown size are immediately apparent, in part because of the much larger samples at each set of gestational extremes. Thus 157 high-birth-weight boys and girls (solid lines) are quite systematically larger-toothed than 178 low birth-weight children (the latter designated here by dashed lines). For the high birth-weight grouping, comprising children in 


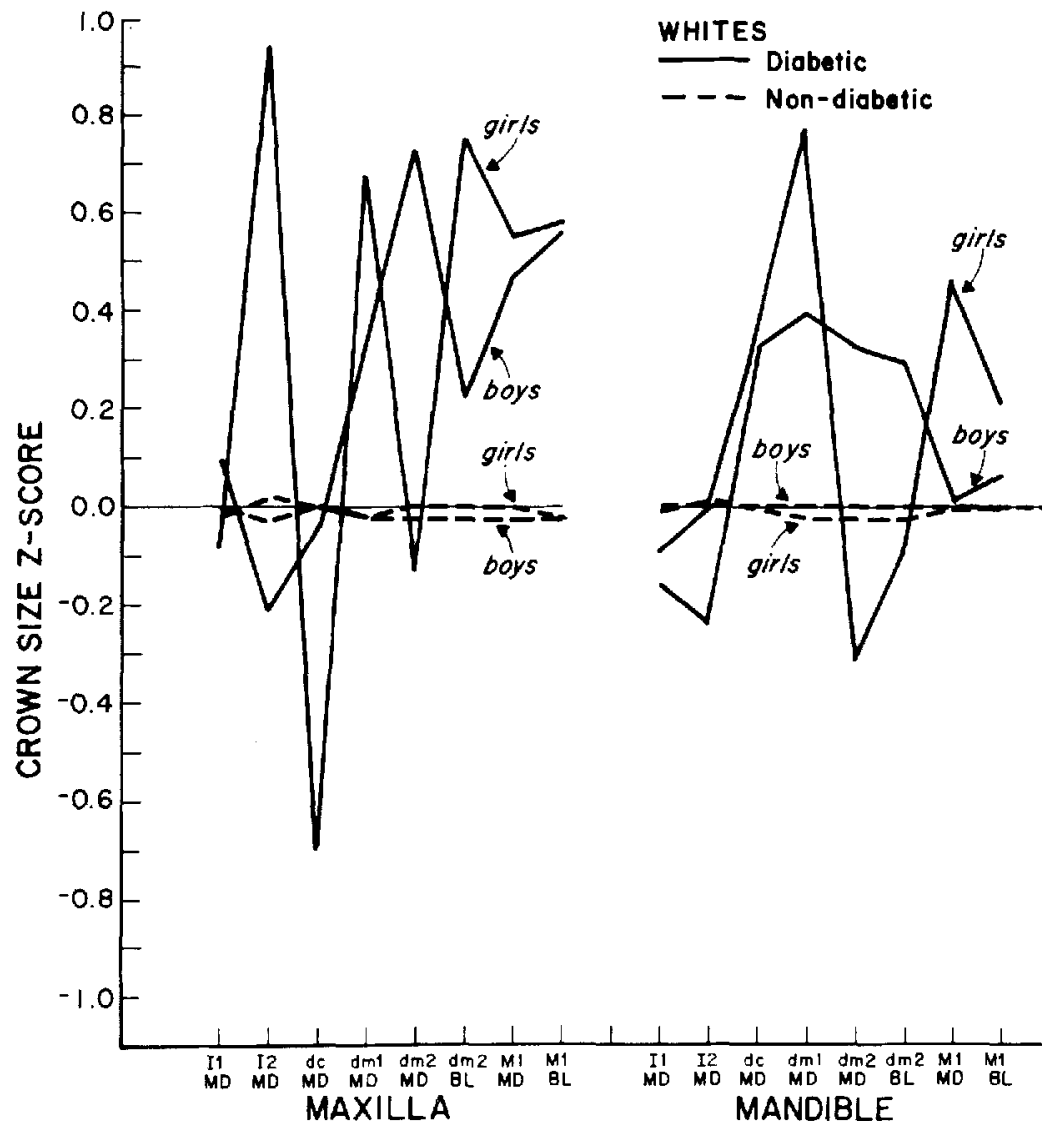

Fig. 3 Crown-size pattern-prafiles of 20 diabetic children in the NCPP sample showing generally-elevated mesiodistal and buccolingual crown sizes for both deciduous and permanent teeth. Analysis of birth sizes of these 20 children does not explain the increased crown dimensions either in terms of greater weight or crown-heel length.

the upper percentiles for weight at birth, there is again a tendency for the boys to be more affected, i.e., showing greater mesiodistal and buccolingual crown size expansion.

In figure 5, contrasting 133 high birthlength and 205 low birth-length children, an effect of birth length on tooth size is again associated with a tendency for the boys to be more affected at both dimensional extremes. The bigger or longer boys are systematically bigger-toothed while the smaller (shorter) boys show the greater association between birth length and crown sizes. However viewed, whether from the standpoint of weight at birth or the partially-independent crown-heel dimension, big babies go with larger deciduous and permanent teeth and little babies with diminished mesiodistal and buccolingual crownsize dimensions. This might appear reasonable if teeth completed their crown development prior to birth, but several of the deciduous and all of the permanent teeth considered in these graphs and tables are characterized by continuing enamel accretion after birth.

The last figure (fig. 6) presents profiled data on dental dimensions for 23 children of hypertensive mothers, again for whites only because of sample size restrictions, and because of population differences in the prevalence of essential hypertension. And the crown-size patterns in this figure show the general tendency for crown-size reduction previously documented as well as the somewhat erratic nature of the patterns in this numerically small sample.

Taking all of the odontometric data into consideration, the mesiodistal and buccolingual raw-score values, the sex-specific and 


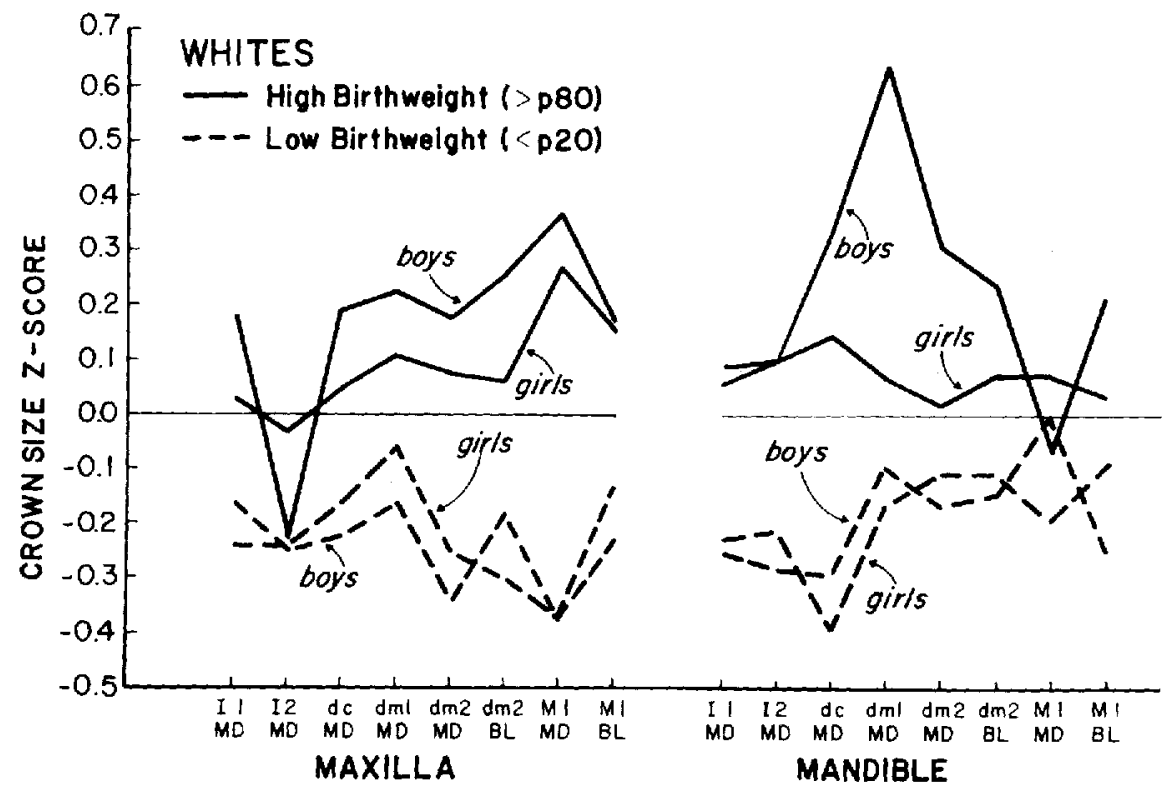

Fig. 4 Effect of birth weight on mesiodistal and buccolingual crown dimensions. As shown, high-birth weight boys and girls (solid lines) differ systematically form low-birth weight boys and girls (dashed lines) in both mesiodistal and buccolingual maxillary and mandibular crown dimensions.

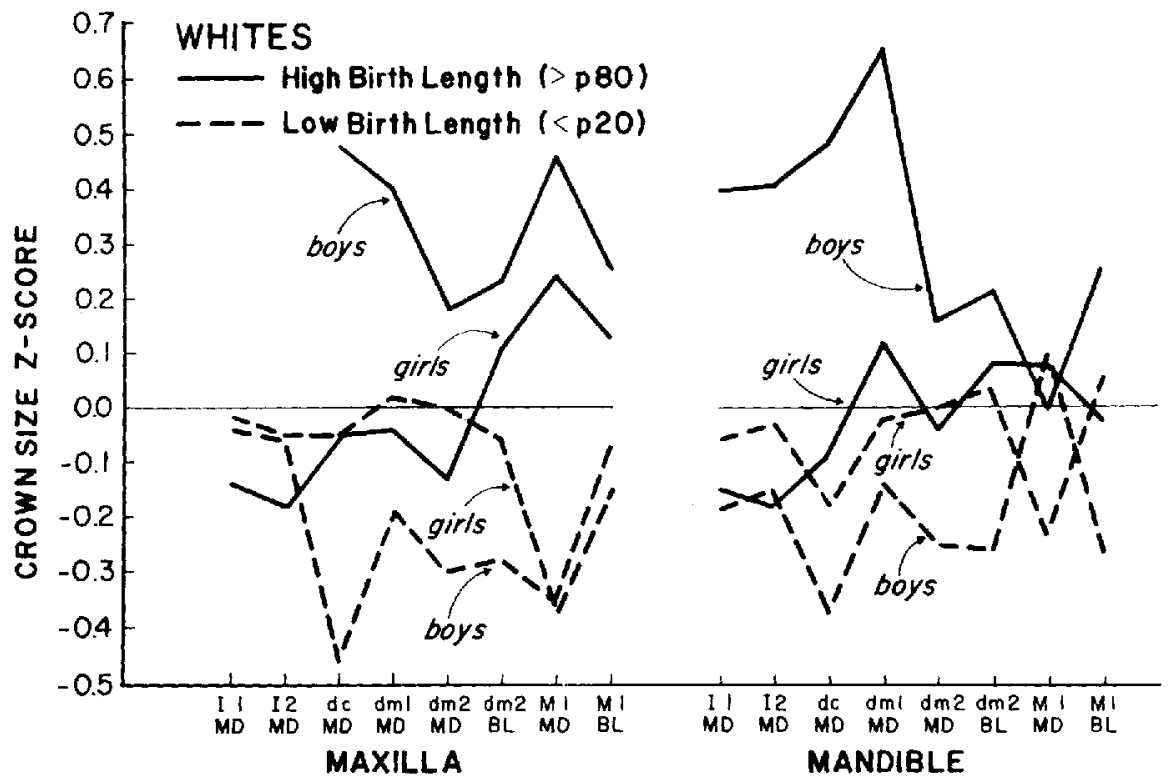

Fig. 5 Relationship between birth length and mesiodistal and buccolingual crown size dimensions. As shown, boys and girls in the upper percentiles of birth lengths (solid lines) tend to larger crown diameters than boys and girls in the lower percentiles of birth length (dashed lines). With the relatively large samples (133 and 205) the systematic odontometric differences are highly significant by serial $t$-tests. 


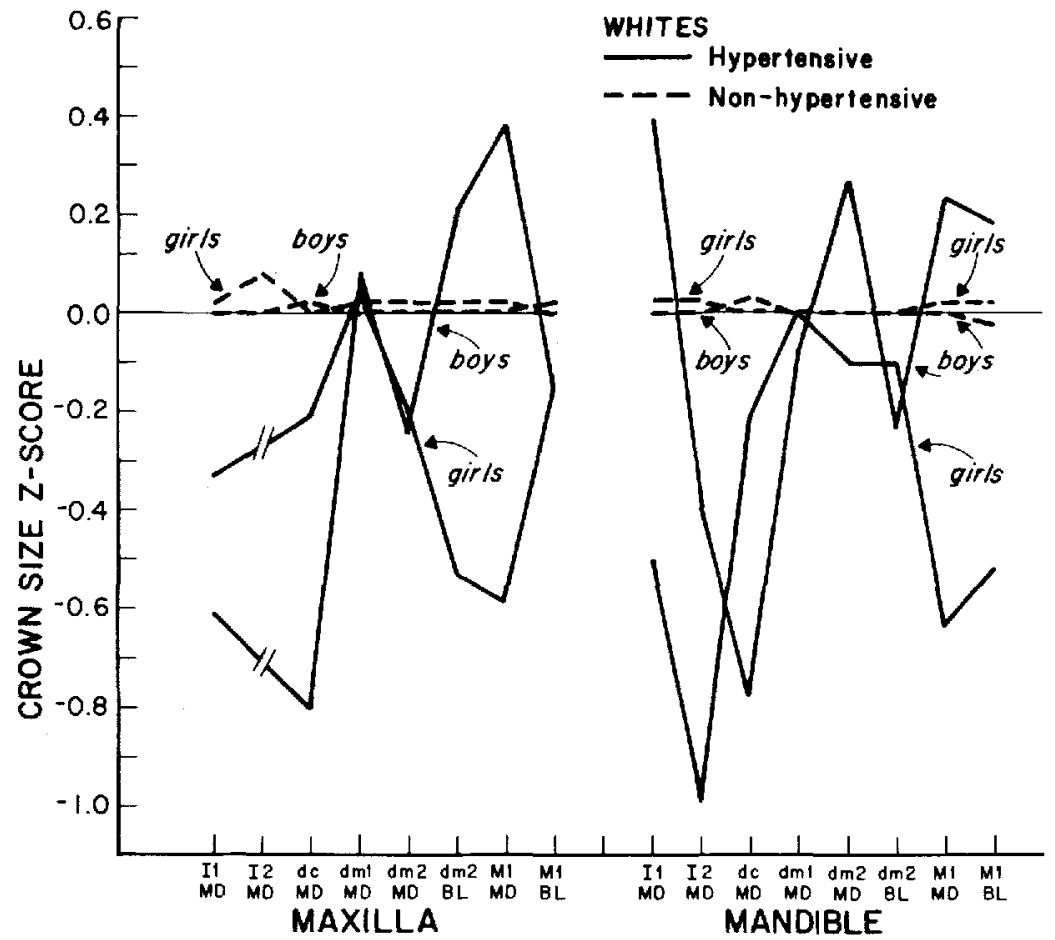

Fig. 6 Effect of maternal hypertension during pregnancy on crown size dimensions of the progeny. As shown here, for 23 such children, crown sizes tend to be diminished in both sexes as compared with the remaining sample (dashed lines) and the total sample (indicated by the reference line). Though marginally larger at birth than the total sample the hypertensive progeny are not significantly different and therefore the gestational variable does not account for the dental diminution.

combined-sex Z-score values and the various graphic summarizations, the trends originally suggested in figure 1 are clear enough for the white children contained within the NCPP series, and with surprisingly good agreement. Maternal health status during gestation does make a difference to tooth size. So does the size of the infant at birth, perhaps in more systematic fashion (and possibly because of sample size). Factors that apparently increase both deciduous and permanent crowns include maternal diabetes, maternal hypothyroidism and large size at birth. Factors that apparently diminish dental dimensions are (1) maternal hypertension, (2) reduced birth weight and (3) diminished crown-heel length.

This hierarchy of maternal and gestational influences on mesiodistal and buccolingual crown-size dimensions demonstrably crosses the occlusal line, affecting both mandibular and maxillary teeth, and the developmental sequence, affecting both deciduous and permanent teeth alike. It also affects different morphological classes-incisors, canines and molars, though with possibly more influence on the lateral (second) incisors. Maternal diabetes, maternal hypothyroidism and the other maternal and gestational conditions also affect both crown dimensions in both sexes, though possibly affecting the crown dimensions of girls less than those of boys.

\section{DISCUSSION}

It should now be evident that a variety of definable factors operating well prior to birth may affect crown dimensions of both deciduous and permanent teeth in both sexes. This demonstration based on a unique collection of odontometric data from a prospective long-term longitudinal growth study has major implications to all odontometric comparisons whether of individuals still to be born or those long-deceased and of the fossil past. In addition to the genetic component of crown size the newly-demonstrated environmental component may together account for a sub- 
stantial proportion of crown-size variance in man.

To begin with, these findings reiterate the importance of the embryonic and fetal periods even for such structures as the crowns of teeth that are completed during postnatal time. To be sure, the permanent teeth attain their definitive crown dimensions after birth, yet the findings in this study indicate that maternal health status during pregnancy and the rate of fetal development together affect crown diameters. Deciduous teeth, of course, attain most of their crown dimensions in the prenatal period. Yet, in the maternal and gestational conditions we have described here, both sets of teeth are altered and often to a comparable degree. This discovery is not unexpected in view of the developmental correlations among the teeth in prenatal time (Garn et al., '75, '78) but it may come as a surprise to those who rely exclusively on radiographic standards of tooth formation and crown completion.

To be sure, there are very real questions as to control mechanisms in each of the "maternal" and "fetal" conditions identified for comparison of mesiodistal and buccolingual crown diameters. In the case of the diabetic progeny (experiencing maternal diabetes during the embryonic period) there is a wealth of evidence pointing to greater prenatal growth rates as we have demonstrated, through the medium of radiographs, many years ago (Garn et al., '56). As a guess, the teeth also participate in this augmented growth of the diabetic fetus. Again, in maternal hypothyroidism there is clinical evidence of compensatory fetal hyperthyroidism. Presumably it is the effect of increased fetal T.S.H. (thyroid-stimulating hormone) and T.H. (thyroid hormone) that we see reflected in the increased crown lengths and breadths.

Effects of gestation length and of size at birth, however, do not engender easy answers or solutions when deciduous and permanent crown sizes are concerned. There is as yet no simple explanation for why a longer gestation length should be associated with larger tooth crowns or why a shorter gestation length might be associated with small odontometric measurements. Indeed, in the past, it was assumed that smaller crowns in dysmorphogenesis states were a function of the developmental abnormality, and not restricted gestation length or diminished size at birth.
Nor is it functionally sufficient to divide the several conditions here considered into a "maternal" group (including hypothyroidism, diabetes, hypertension, etc.) and a "fetal" group - prematurity/postmaturity, birth size etc. As we have otherwise shown, a great many maternal conditions affect the size of the fetus and the newborn, and continue to have demonstrable effects as late as the seventh year. Smoking mothers tend to have small newborns, and these small infants remain small for years to come (Garn et al., '77, '78). Obese mothers engender larger placentas (Garn et al., '79) the larger placentas are associated with larger neonates and the larger neonates of obese mothers are larger still at seven years.

Depending upon the area of interest there are many disturbing implications to these findings to population studies and demographic research. If small size at birth is associated with diminished crown size, then some part of the apparent trend to smaller odontometric dimensions may derive from the improved survival of small and developmentally-immature neonates. If large permanent and deciduous teeth are associated with large size at birth, then there may be an odontometric effect of induced labor and attempts to minimize intrauterine fetal growth. Indeed, deliberate attempts to alter fetal weight gain during pregnancy may disrupt the "normal" range of crown-size diameters, scarcely speculation we might add.

There is the question of prenatal mortality where prenatal care is less effective, and the effects of prenatal wastage and loss on the crown-size continuum as we know it. There is the further question as to whether differential loss of premature infants, small-for-date infants and the progeny of small and lowweight mothers together affect postnatal tooth dimensions by selectively removing the neonates with smaller teeth. Indeed there is the further question of whether conventional twin correlations are artificially augmented by including low-weight, premature and small-for-date multiple births.

Yet a caution is indicated. These new findings should not be over-interpreted. While they are systematic and consistent they are subject to sampling errors and any one of these new findings may fail to be replicated in a larger and independent sample. Moreover, it may not be diabetes that directly makes for 
larger crown size of the offspring but the level of maternal nutrients available to the fetus or compensatory fetal hyperinsulinism, or both. Small neonatal size may reflect a deficiency at the maternal side of the placenta, or growth failure at the fetal side or both.

Still, this study retains its major value. It demonstrates that prenatal factors may affect crown size during fetal development and therefore the mesiodistal and buccolingual dimensions we customarily measure. Without question it casts a new and penetrating light on all the measurements we have so far collected and in all the interpretations so far made. Population differences, historical changes, geographical "clines" and even family-line resemblances in the classical crown dimensions may, to a greater or lesser extent, simply reflect systematic prenatal influences on the crown lengths and crown breadths of the developing teeth. Taking the extreme conditions of maternal hypothyroidism and maternal hypertension both into consideration, possibly half of crown size variability may thus be explained.

\section{ACKNOWLEDGMENTS}

The work described in this paper was supported, in part, by contract N01-NS-2-2302 from the National Institute of Neurological Disorders and Stroke to the University of Wisconsin and contract N01-NS-5-2308 to the University of Michigan. Further support came from Grant DE 03443 from the National Institute of Dental Research and Grant DE 03610 , both to the University of Michigan. The careful assistance of Sirka Alvesalo, Helen Bennett and Gisela Naas in the odontometric phase at the University of Wisconsin was responsible for the data quality as was the programming assistance of Mr. Russell Spry. At the University of Michigan Center for Human Growth we are indebted to Mr. Robert Wainright for programming assistance, $\mathrm{Mr}$. Arthur Ensroth for major involvement of the data analysis and Karin Hoff in completing the study. The manuscript was completed by Pamela G. Reed.

\section{LITERATURE CITED}

Abramowitz, M., and I. A. Stegun 1970 Handbook of Math- ematical Functions. National Bureau of Standards, U. S. Dept. of Commerce, Washington.

Bailit, H. L., and B. Sung 1968 Maternal effects on the developing dentition. Archs. Oral. Biol., 13: 155-161.

Burdi, A. R., S. M. Garn and J. Superstine 1975 Correlates of permanent tooth developmet in prenatal time. J. Dent. Res., 54: 697

Cohen, M. M., B. J. Baum, S. M. Garn, C. H. Osorio and J. M. Nagy 1977 Crown-size reduction in congenital defects. In: Orofacial Growth and Development. A. A. Dahlberg and T. M. Graber, eds. Mouton Publishers, The Hague, pp. $119-126$

Cohen, M. M., S. M. Garn and M. A. Geciauskas 1970 Crown size profile pattern in trisomy. J. Dent. Res., 49: 460.

Dijkman, D. J, 1978 Patterns of Mesiodistal Tooth Size Alteration. M. A. thesis, University of Toronto.

Evans, M. W. 1944 Congenital dental defects in infants subsequent to maternal rubella during pregnancy. Med. J. Austral., 31: 225-228.

1947 Further observations on dental defects in infants subsequent to maternal rubella during pregnancy. Med. J. Austral., 1: 780-785.

Garn, S. M. 1977 Genetics of dental development. In: The Biology of Occlusal Development. J. A. McNamara, ed. Center for Human Growth, Ann Arbor, Michigan.

Garn, S. M., and S. M. Bailey 1977 The symmetrical nature of bilateral asymmetry ( $\delta$ ) of deciduous and permanent teeth. J. Dent. Res., 56: 1422

Garn, S. M., A. R. Burdi, W. J. Babler and R. Asp 1979 Crown size-arch space relationships during human prenatal dental development. J. Dent. Res., 58:554-559.

Garn, S. M., A. R. Burdi and J. Superstine 1975 Early prenatal variability in the order of permanent tooth development. J. Dent. Res., 54: 420 .

Garn, S. M., M. M. Cohen, J. C. Gall and J. Nagy 1971 Relative magnitudes of crown size reduction. In: 47-Trisomy G. J. Dent. Res., 50: 513

Garn, S. M., M. M. Cohen and M. A. Grekiauskas 1970 Increased crown-size. In: Trisomy. G. J. Dent. Res., 49: 465

Garn, S. M., G. R. Greaney and R. W. Young 1956 Fat thickness and growth progress during infancy. Hum. Biol., 28: $232-250$

Garn, S. M., K. Hoff and K. D. McCabe 1979 Maternal fatness and placental size. Am. J. Clin. Nutr., 32:277-279.

Garn. S. M., H. A. Shaw and K. D. MeCabe 1977 Effects of socioeconomic status and race on weight-defined and gestational prematurity in the United States. In: The Epidemiology of Prematurity. D. Reed and F. Stanley, eds. Urban and Schwarzenberg, Baltimore.

Garn, S. M., H. A. Shaw and K. D. McCabe 1978 No ifs, ands or butts: an essay on the maternal smoking effect. In Symposium on Human Nutrition. R. Schemmel, ed. Research Report 364, Michigan State University.

Moyers, R. E., F. P. G. M. van der Linden, M. L. Riolo and J. A. McNamara 1976 Standards of Human Occlusal Development. The University of Michigan, Ann Arbor.

Niswander, K. R., and M. Gordon 1972 The Women and Their Pregnancies. W. B. Saunders, Philadelphia.

Osorio, C. H. 1970 Variations in the Mesiodistal Coronal Diameter of Permanent Teeth in Individuals with Oral Clefts. Unpublished Masters thesis, University of Michigan, Ann Arbor. 